\title{
Experiencia de innovación docente en la asignatura de Cuencas Hidrográficas y Gestión del Agua (Geografía Física)
}

BelÉn GaRcía MaRTínez

Universidad de Sevilla

Dpto. Geografía Física y Análisis

Geográfico Regional

mbelen@us.es

ORCID: https://orcid.org/0000-0002-4589-9385

D.O.I.: http://dx.doi.org/10.12795/JDU.2018.i01.92

Pp.: $1641-1656$

\section{Resumen}

Se presentan los primeros resultados sobre la experiencia de innovación docente en la asignatura de Cuencas Hidrográficas y Gestión del Agua de la titulación de Geografía y Gestión del Territorio. La implementación de un Ciclo de Mejora Docente (CMD) a partir de la definición de un modelo metodológico posible, basado en problemas y centrado en el aprendizaje del alumno, pone de manifiesto una progresiva vinculación del estudiante con su propio aprendizaje conforme va generando conocimiento a partir de la investigación. Asimismo, supone un cambio en el modelo didáctico transmisivo que da paso a otro modelo más integrador del que participa los contenidos, la metodología y la evaluación del aprendizaje. 
Palabras clave: Cuencas hidrográficas y gestión del agua, Geografía y Gestión del Territorio, docencia universitaria, experimentación docente universitaria

\section{Contexto}

La asignatura en la que se lleva a cabo esta experiencia es Cuencas hidrográficas y gestión del agua, optativa de cuarto curso del Grado de Geografía y Gestión del Territorio y del Doble Grado de Geografía y Gestión del Territorio e Historia. Esta asignatura, de 6 créditos, es impartida por dos áreas de conocimientos, Geografía Física y Geografía Humana, con una asignación de 4 y 2 créditos respectivamente. Los créditos asignados al área de Geografía Física corresponden a contenidos teórico-prácticos $(2,5)$ y a contenidos prácticos en el campo (1,5); aplicándose el ciclo de mejora en los contenidos teórico-prácticos. Esta asignatura optativa cuenta con una matrícula de 17 alumnos para el curso 2018-2019 (8 alumnos de Grado y 9 de Doble Grado), de los cuales, 16 se comprometieron a participar en esta experiencia de aprendizaje. Las clases son de hora y media de duración en un aula sin ventanas y con bancas estáticas, lo que dificulta el trabajo en grupo.

\section{Diseño del Ciclo de Mejora}

\section{Mapa de Contenidos}

El mapa de contenidos que se presenta (figura 1) hace referencia a un apartado del temario correspondiente al análisis de parámetros hidrológicos y morfométricos que caracterizan el comportamiento hidrológico natural de una cuenca. En ese contexto, se individualizan los contenidos que van a ser abordados en el Segundo Ciclo de Mejora Docente. 


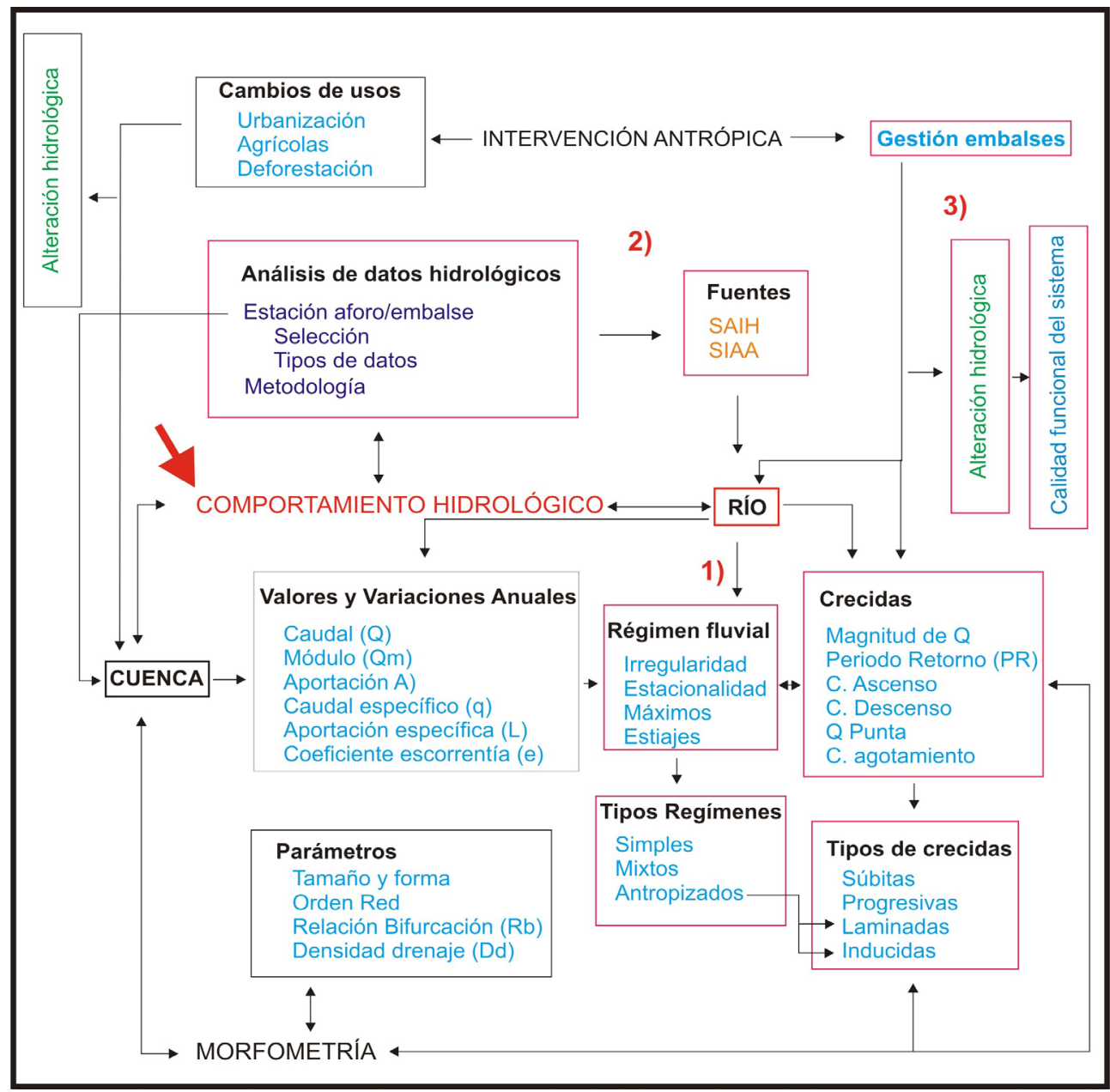

\section{Comienzo
Segundo ciclo de mejora \\ Segundo ciclo de mejora \\ Contenidos aprendizaje 2CMD}

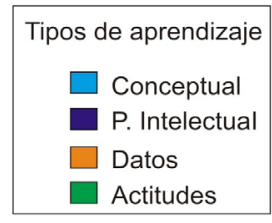
1), 2) y 3) Contenidos de aprendizaje sobre los problemas concretos planteados (Cuestionario)

\section{CASO/PROBLEMA}

Existe un grave problema de bastecimiento hídrico en la ciudad de Sevilla, y EMASESA (Empresa de Abastecimiento y Saneamiento de Aguas de Sevilla $S A$ ) nos pide que evaluemos cuál podría ser el sistema fluvial más idóneo, desde un punto de vista hidrológico, que pudiera solventar el problema de abastecimiento en la capital andaluza.

Figura 1. Mapa de contenidos: Parámetros hidrológicos y morfométricos para la caracterización hidrológica natural de una cuenca.

Jornadas de Formación e Innovación Docente del Profesorado | № 1 (2018)

(c) (i) $\odot$ Esta obra se distribuye con la licencia Creative Commons 
Los contenidos que se trabajan en este ciclo son los correspondientes a dos de los modelos de escorrentía que pueden analizarse en un río a nivel de cuenca, esto es: el régimen fluvial y las crecidas fluviales. Para ello, el alumno habrá de enfrentarse a conceptos teóricos y prácticos, así como al problema existente en las fuentes de datos disponibles. Con todo ello, el alumno irá construyendo el aprendizaje hasta determinar de qué manera una actuación antrópica como la gestión de embalses puede llegar a alterar el comportamiento natural de una cuenca, lo que influirá en la calidad funcional de su sistema; y del que depende innumerables elementos (funcionalidad de la llanura, naturalidad del trazado, continuidad de los procesos longitudinales y verticales, naturalidad de las márgenes y de la movilidad lateral, continuidad longitudinal de las riberas, anchura del corredor ribereño, o estructura, naturalidad y conectividad trasversal de las riberas), sobre los que trabajaremos hasta final del cuatrimestre.

La construcción del mapa de contenidos ha supuesto una profunda reflexión no sólo sobre los contenidos que articulan el aprendizaje y sus relaciones, destruyendo, en parte, los departamentos estancos que infiere la distribución por temas; sino también sobre otras cuestiones, que si bien habían estado presentes, nunca las había formulado conscientemente, como parte del aprendizaje de esta asignatura. Me refiero tanto a cuestiones sociales o éticas relacionadas con la materia, como a la distinción de los tres grupos básicos de contenidos, esto es, conceptuales, procedimentales y actitudinales (García Díaz et al., 2017).

Hay que saber que cada alumno, antes de llegar a este segundo ciclo de mejora, ha tenido que seleccionar un sistema fluvial (con el único criterio de que hubiera disponibilidad de datos) y definirle su cuenca. Este paso supone un paso importante para el estudiante ya que supone enfrentarse a conceptos teórico-prácticos sobre la interpretación del relieve, totalmente novedoso, y que suponen una habilidad intelectual que han de ir desarrollando. Asimismo, se hace constar que cada uno ha elegido un sistema fluvial 
diferente, de cara a valorar al final de curso cuál es el sistema fluvial más idóneo para abastecer a la ciudad de Sevilla. Es por ello, por lo que la tutoría adquiere un valor importante para ir contrastando con el alumno los pasos realizados y que no hayan podido resolverse en el aula.

\section{Modelo Metodológico}

La formulación del modelo metodológico posible (figura 2), superadas las dificultades contextuales que condicionan nuestra labor docente (Porlan et al., 2010), ha supuesto un cambio en la tarea docente y también un cambio en la tarea de aprendizaje del alumno. A este respecto, se introduce parte del modelo aprender investigando (Feria, 2017), de cara a que el alumno se implique y comprometa en su tarea de aprendizaje.

El modelo metodológico posible, parte del planteamiento de un problema sobre el que el alumno ha de plantear sus ideas iniciales. A partir de ellas, y mediante actividades de contraste teórico-prácticas, el alumno ha de reformularlas para la resolución del problema. Es modelo busca crear un entorno apropiado para el aprendizaje crítico natural, otorgando al estudiante la libertad para que aborden cuestiones y tareas sobre las que tomar decisiones, reciban retroalimentación de sus hipótesis o ideas y prueben de nuevo (Bain, 2004).

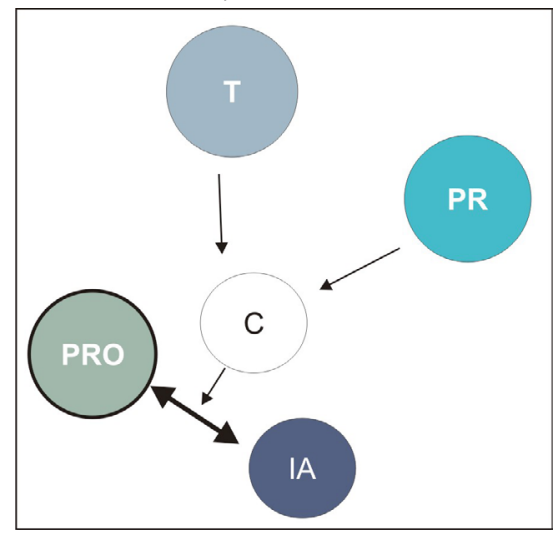

Figura 2. Modelo metodológico posible

Jornadas de Formación e Innovación Docente del Profesorado I № 1 (2018) Esta obra se distribuye con la licencia Creative Commons 
a) Día 30 de 0ctubre

\begin{tabular}{|l|l|l|}
\hline 1 & IA & $15^{\prime}$ \\
\hline \multicolumn{2}{|c|}{ Modelos de escorrentía } \\
¿Qué importancia tiene la escala espacial y temporal en el \\
comportamiento hidrológico de un sistema fluvial? \\
$\begin{array}{l}\text { El alumno tiene que tratar de responder, de manera razonada, a esta } \\
\text { cuestión a partir de sus creencias }\end{array}$ \\
\hline Recursos \\
Cuaderno de trabajo \\
\hline
\end{tabular}

\begin{tabular}{|c|c|c|}
\hline 3 & IA(reformulada) & $30^{\prime}$ \\
\hline \multicolumn{3}{|c|}{$\begin{array}{l}\text { Modelos de escorrentía } \\
\text { ¿Qué importancia tiene la escala espacial y temporal en el } \\
\text { comportamiento hidrológico de un sistema fluvial? }\end{array}$} \\
\hline \multicolumn{3}{|c|}{$\begin{array}{l}\text { En primer lugar, cada alumno caracteriza su cuenca a nivel espacial } \\
\text { (previo a este ciclo de mejora, el alumno ha tenido que seleccionar } \\
\text { un sistema fluvial, a partir de los criterios definidos por la profesora, y } \\
\text { delimitar su cuenca). }\end{array}$} \\
\hline \multicolumn{3}{|c|}{$\begin{array}{l}\text { En segundo lugar, el alumno plantea una hipótesis de trabajo sobre e } \\
\text { comportamiento hidrológico del río seleccionado: ¿régimen fluvial? } \\
\text { ¿tipo de escorrentía de la cuenca? ¿Variabilidad interanual? } \\
\text { ¿Crecidas? ¿estiajes? Todo ello a partir de la localización geográfica }\end{array}$} \\
\hline \multicolumn{3}{|c|}{$\begin{array}{l}\text { Por último, el alumno describe que tipo de datos necesitará en cada } \\
\text { caso para analizar los distintos modelos de escorrentia. Explica las } \\
\text { razones }\end{array}$} \\
\hline \multicolumn{3}{|c|}{ Recursos } \\
\hline \multicolumn{3}{|c|}{$\begin{array}{l}\text { Cuenca delimitada (archivos shp. ArcGis) } \\
\text { Documento de contraste } \\
\text { Mapa Cuenca Hidrográfica del Guadalquivir } \\
\text { Cuaderno de trabajo }\end{array}$} \\
\hline
\end{tabular}

\begin{tabular}{|c|c|c|c|}
\hline 2 & $2 \mathrm{CMD}$ & CONTRASTE & $20^{\prime}$ \\
\hline \multicolumn{4}{|c|}{$\begin{array}{l}\text { 1. Se le aporta al estudiante el epígrafe de un capítulo que aborda la } \\
\text { necesidad de tipificación de las aguas fluviales en función de } \\
\text { la escala espacial o temporal: } \\
\text { a) Escala espacial: el tamaño de la cuenca es la variable más } \\
\text { importante en la tipificación de las aguas. } \\
\text { b) Escala temporal: } \\
\text { *Valores medios mensuales (serie de } 30 \text { años), esto es el } \\
\text { régimen } \\
\text { * Escorrentia total media que produce una cuenca y } \\
\text { variaciones anuales de la escorrentia } \\
\text { * Caudales máximos absolutos (crecidas) y mínimos (estiaje) }\end{array}$} \\
\hline \multicolumn{4}{|c|}{$\begin{array}{l}\text { Sala Sanjaume, M. (1989). Aguas Continentales, En Vielza de Ory (coord): Territorio y } \\
\text { Sociedad en Espala I. Geografia Fisica, pp.275-277 }\end{array}$} \\
\hline 4 & $2 \mathrm{CMD}$ & CONTRASTE & $25^{\prime}$ \\
\hline \multicolumn{4}{|c|}{$\begin{array}{l}\text { Problemas de las fuentes } \\
\text { 1. SAlH Guadalquivir (Sistema Automático de Información Hidrológica) } \\
\text { 2. SIAA (Sistema de Información de Anuarios de Aforos) } \\
\text { 3. Tipos y calidad de los datos }\end{array}$} \\
\hline $\begin{array}{l}\text { Recur } \\
\text { hitp:/// } \\
\text { https:/ } \\
\text { inform }\end{array}$ & $\begin{array}{l}\text { guadalo } \\
\text { iteco.g } \\
\text { nuario- }\end{array}$ & /temas/evaluacion-de & istema- \\
\hline
\end{tabular}

Figura 3. Secuencia de actividades sesión 1 


\section{b) Día 5 de noviembre}

\begin{tabular}{|l|l|l|}
\hline 5 & 2 IA & 10 \\
\hline \multicolumn{2}{|c|}{$\begin{array}{c}\text { ¿Qué entiendes por régimen fluvial? } \\
\text { ¿Qué aporta al estudio hidrológico de un sistema fluvial? } \\
\text { ¿Cómo crees que se obtiene? }\end{array}$} \\
$\begin{array}{l}\text { Recursos } \\
\text { Cuaderno de trabajo }\end{array}$ \\
\hline
\end{tabular}

\begin{tabular}{|c|c|c|c|}
\hline 6 & $2 \mathrm{CMD}$ & CONTRASTE & 30 \\
\hline \multicolumn{4}{|c|}{ ¿Cómo se calcula y describe el régimen fluvial? } \\
\hline \multicolumn{4}{|c|}{$\begin{array}{l}\text { El régimen fluvial como modelo teórico de comportamiento se analiza } \\
\text { mediante el coeficiente de caudal, que no es más que la relación entre } \\
\text { el caudal medio de un mes determinado y el caudal modular de la serie. }\end{array}$} \\
\hline \multicolumn{4}{|c|}{$\mathrm{Cq}=\mathrm{Qmm} / \mathrm{Qm}$} \\
\hline \multicolumn{4}{|c|}{$\begin{array}{l}\text { De esta manera, la frecuencias relativa de los caudales mensuales en una } \\
\text { serie de } n \text { años, permite poner en evidencia el carácter sistémico de las } \\
\text { variaciones estacionales y la evolución de su comportamiento en la serie; } \\
\text { pudiendo definirse cambios en el régimen fluvial. }\end{array}$} \\
\hline \multicolumn{4}{|c|}{$\begin{array}{l}\text { En él queda representado tanto el periodo de aguas altas, integrado por } \\
\text { aquellos meses con caudales medios superiores al módulo }(\mathrm{Cq}>1) \text {, como } \\
\text { los máximos estacionales y el periodo de guas bajas, con caudales medios } \\
\text { inferiores al módulo (Cq<1) y en el que puede llegar a identificarse estiajes, } \\
\text { para meses en los que el coeficiente de caudal se aproxime a cero. }\end{array}$} \\
\hline \multicolumn{4}{|c|}{ Recursos } \\
\hline \multicolumn{4}{|c|}{ Pizarra } \\
\hline
\end{tabular}

\begin{tabular}{|l|l|c|}
\hline 7 & \multicolumn{1}{|c|}{ CONTRASTE } & $20^{\circ}$ \\
\hline \multicolumn{1}{|c|}{ Régimen Fluvial } \\
\\
Se aporta al alumno referencias bibliográficas sobre Regimenes \\
fluviales. \\
En ellas se pone de manifiesto la clasificación tradicional sobre \\
regímenes fluviales asi como la problemática que existe actualmente \\
para su utilización
\end{tabular}

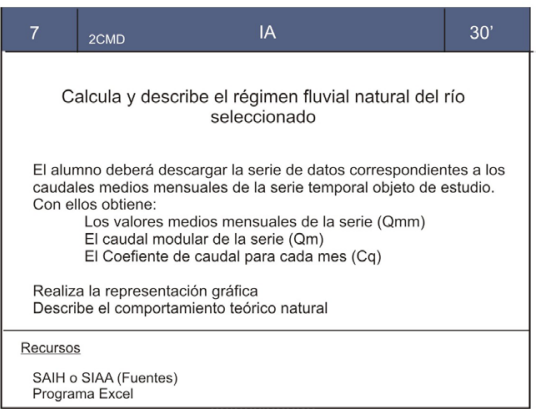

Figura 4. Secuencia de actividades de la sesión 2

\section{c) Día 6 de noviembre}

\begin{tabular}{|l|l|l|}
\hline 8 & IA & 15 \\
\hline \multicolumn{3}{|c|}{$\begin{array}{l}\text { ¿QMD } \\
\text { ¿Qué entiendes por crecida? } \\
\text { ¿Cómo se estudia hidrológicamente? } \\
\text { ¿Podrías diferenciar tipos de crecidas? }\end{array}$} \\
\hline $\begin{array}{l}\text { Recursos } \\
\text { Cuaderno de trabajo }\end{array}$ \\
\hline
\end{tabular}

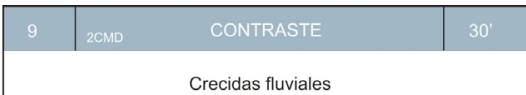

En clase se explica al estudiante y se aporta bibliografia sobre:

"El concepto de crecida y diferenciación de otros conceptos como avenida, desbordamiento, riada e inundación. "Factores condicionantes de una crecida (tipo de cuenca. tipo de red,

(progresivas), de origen convectiva (súbitas), de origen antrópico (laminadas Recursos

Power-point sobre crecidas fluviales a partir de la bibliografia aportada Mateu. J.F. (1989): Crecidas e inundaciones, Guia de la Naturaleza de la
Mation Comunidad valenciana, pp. 568-608

Camarasa Belmonte, A.M. (2002): Crecidas e inundaciones, En Ayala Carcedo y Gil Olcina (Coords): Riesgos Naturales, Ed. Ariel, pp.859-869 ( 


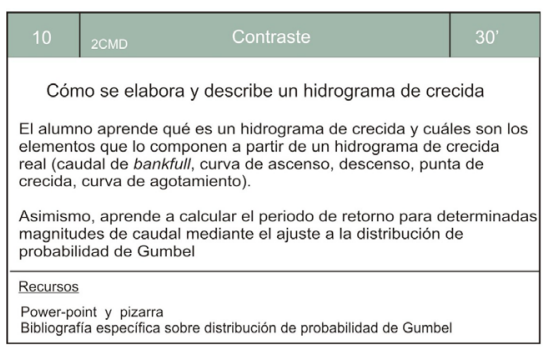

\begin{tabular}{|c|c|c|c|}
\hline 11 & $2 C M D$ & IA (entre clases) & $60^{\prime}$ \\
\hline \multicolumn{4}{|c|}{$\begin{array}{l}\text { Elabora y describe el hidrograma de crecida natural } \\
\text { del sistema fluvial seleccionado }\end{array}$} \\
\hline \multicolumn{4}{|c|}{$\begin{array}{l}\text { El alumno descarga los datos correspondientes a los eventos de crecida } \\
\text { de su sistema fluvial (datos diarios) y construye sus correspondientes } \\
\text { hidrogramas. Descompone el hidrograma y describe el comportamiento } \\
\text { de la onda de crecida. }\end{array}$} \\
\hline \multicolumn{4}{|c|}{$\begin{array}{l}\text { El alumno descarga el caudal máximo diario anual de la serie estudiada } \\
\text { y calcula la magnitud de caudal para periodos de recurrencia de } 5,10 \text { y } \\
25 \text { años (según distribución Gumbel). }\end{array}$} \\
\hline \multicolumn{4}{|c|}{ Recursos } \\
\hline \multicolumn{4}{|c|}{$\begin{array}{l}\text { Cuaderno de trabajo } \\
\text { Bibliografia especifica } \\
\text { SAlH Guadalquivir } \\
\text { Programa Excel }\end{array}$} \\
\hline
\end{tabular}

Figura 5 (a y b). Secuencia de actividades de la sesión 3

\section{d) Día 12 de noviembre}

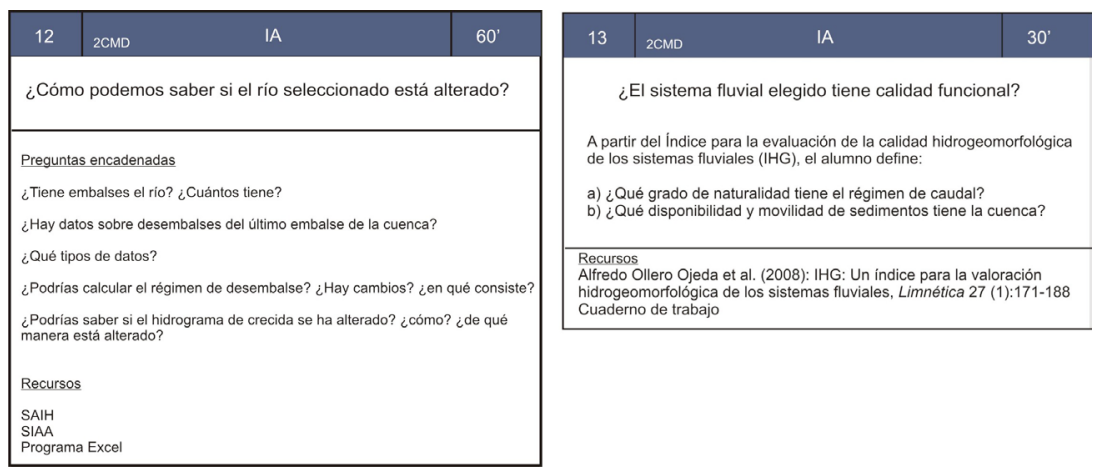

Figura 6. Secuencia de actividades de la sesión 4

\section{Cuestionario}

El cuestionario se elabora en base al mapa de contenido y al problema o caso planteado, sobre el que se tiene que investigar y dar respuesta. Las preguntas son abiertas e indirectas para que el alumno responda con argumentos (Rivero y Porlán, 2017). Existe un grave problema de abastecimiento hídrico en la ciudad de Sevilla y EMASESA (Empresa de Abastecimiento y Saneamiento de Aguas de Sevilla SA) nos pide que evaluemos cuál sería el sistema fluvial más idóneo, que pudiera abastecer la demanda de agua de la capital andaluza.

1. ¿Cómo ha de ser hidrológicamente el sistema fluvial elegido para que pueda abastecer a la ciudad de Sevilla? 
2. Nos enfrentamos a la problemática de los datos, ¿serían válidos los datos asociados a cualquier estación de aforo para caracterizar hidrológicamente un sistema fluvial? Razona tu respuesta

3. ¿Qué entiendes por calidad funcional del sistema? 3a. ¿Por qué es necesario tener en cuenta la calidad funcional del sistema en la elección del sistema fluvial que abastezca a la ciudad de Sevilla? 3b. ¿Qué argumentos tenemos para decir si es esto una cuestión importante o secundaria?

\section{Aplicación del Ciclo de Mejora Docente}

\section{Sesiones}

Las cuatro sesiones diseñadas inicialmente se tradujeron finalmente en cinco sesiones. Este hecho se debe a la complejidad que conlleva que cada alumno esté generando su aprendizaje a partir de sistemas fluviales diferentes; y, también, a los ritmos de aprendizaje distintos, en algunos casos motivados por la no asistencia a clase de manera regular. A este respecto, de los dieciséis alumnos que empezaron tan sólo doce se han mantenido constantes.

Todas las sesiones comienzan con una batería de preguntas, sobre las que el alumno ha de formular sus ideas y creencias. Esta fase es la que más les cuesta. No están acostumbrados a este tipo de aprendizaje y reflejan inicialmente ciertas reticencias. Tras ello, el docente aporta material de contraste teórico-práctico sobre régimen fluvial y crecidas fluviales, para que puedan reformular sus ideas e hipótesis. Para algunos de los contenidos, como por ejemplo el régimen fluvial, el docente aporta hasta en tres ocasiones material de contraste para que el alumno pueda definir correctamente el régimen de cada sistema fluvial elegido. 
Lo que más les ha constado ha sido la relación que tiene la escala espacial y temporal en el estudio del comportamiento hidrológico, y el acceso a los datos de caudal para llevar a cabo dicho análisis. En este aspecto nos detuvimos más de lo programado, pero era necesario porque de esta fase dependía su investigación posterior. Este hecho provocó que las sesiones siguientes tuvieran que ser reestructuradas y que la reformulación de las hipótesis, programadas para realizarse en el aula, tuvieran que realizarse entre clase y clase. A este respecto hay que decir que ningún alumno tuvo inconveniente en realizar este trabajo en casa.

La implementación del modelo metodológico posible en la secuencia de actividades, me ha permitido quitarme del centro del aula y poner a los estudiantes en contacto directo con el material, tal y como propone Finkel (2000) para dar clase con la boca cerrada.

Por último decir, que si bien el hecho de que cada alumno tuviera un sistema fluvial diferente ha complejizado la labor del docente, teniendo que utilizar las tutorías como extensión de la docencia en el aula; ha sido especialmente motivador para el alumno, en la medida en la que ha ido generando conocimiento a la vez que iba desarrollándose el aprendizaje.

\section{Evaluación del aprendizaje}

La agrupación de las respuestas de los estudiantes en los cuestionarios iniciales y finales, a partir de su grado de complejidad (Duarte, 2015), plantean estas consideraciones:

a) En la primera pregunta más del 75\% de las respuestas se engloban en un nivel de complejidad igual o inferior al C (figura 7). El paso al nivel B o A exige comprender la complejidad sistémica que 
caracteriza a los ríos. Al final del ciclo, el 75\% logran el objetivo.

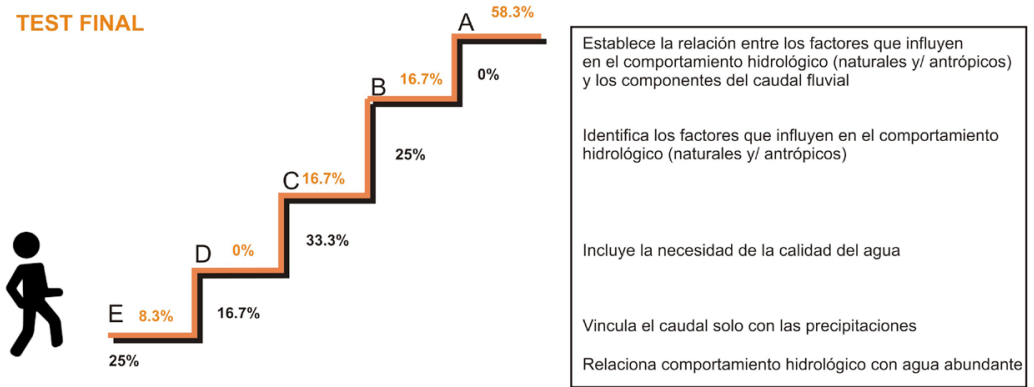

TEST INICIAL

Figura 7. Aprendizaje sobre la pregunta 1

b) En la segunda pregunta, algo más del $40 \%$ de las respuestas o carecen de sentido o contestan sin argumentos (figura 8). En este caso, la mitad de la clase tiene problemas con la componente espacial y temporal en el análisis hidrológico fluvial. Sin embargo, el hecho de que cada alumno tuviera una cuenca diferente de estudio le ha puesto en contacto directo con la problemática e idoneidad de los datos hidrológico durante el análisis. Esto ha influido en que tras el ciclo más del $91 \%$ de los alumnos hayan conseguido el objetivo propuesto.

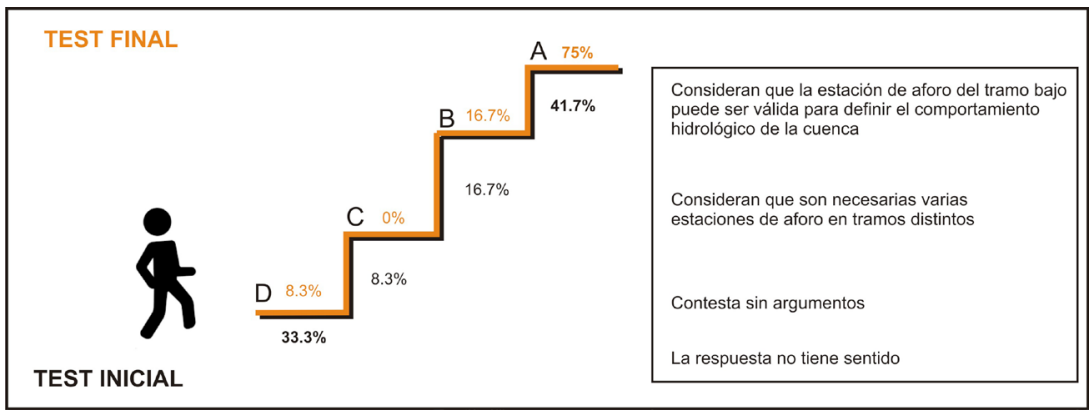

Figura 8. Aprendizaje sobre la pregunta 2

c) En la tercera, el 75\% aportan respuestas simples sobre la calidad funcional del sistema, de las 
cuales algo más del 41\% carecen de sentido o directamente no responden (figura 9). No es extraño si consideramos que la mayoría es la primera vez que trabajan este concepto, por lo que las respuestas se orientan a repetir con las mismas palabras lo que pretenden describir. El aprendizaje implica comprender que la calidad funcional del sistema está asociada a la naturalidad del régimen fluvial, del tránsito sedimentario y de la funcionalidad de las llanuras (Ollero et al., 2008). Tras el análisis comparado que realiza el alumno en su sistema fluvial de los regímenes fluviales y los hidrogramas de crecida en condiciones naturales y antrópicas, más del 75\% aprenden qué significa la calidad funcional del sistema.

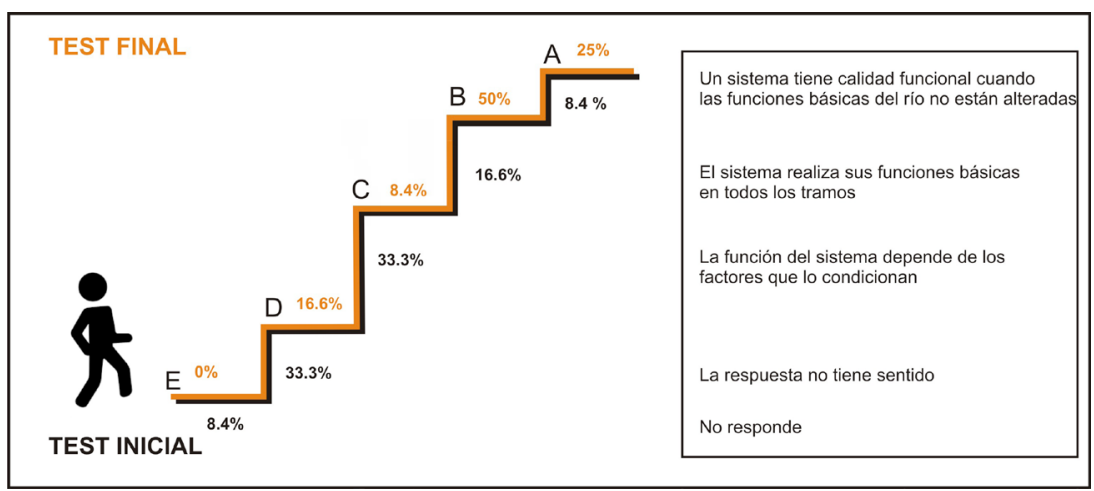

Figura 9. Aprendizaje sobre la pregunta 3

d) Las respuestas a la siguiente pregunta (3a), vinculada a la anterior, muestra de forma evidente (100\%) la falta de comprensión del concepto y por tanto su necesidad como criterio determinante a la hora de elegir al sistema fluvial que abastezca a Sevilla (figura 10). Tras el ciclo, el 83\% de los estudiantes relacionan calidad del agua con calidad del río, es decir, no sólo es 
importante abastecer de agua sino cuidar la dinámica de los sistemas fluviales.

e) En la última pregunta (3b), también relacionada con la tercera, más del $91 \%$ de los alumnos no aportan respuestas con sentido (figura 11). En este caso, los obstáculos son mayores y eso hace que tan sólo algo más del 58\% sea capaz de argumentar respuestas algo más complejas. A este respecto, habrá que seguir profundizando sobre este tema para que todos los alumnos puedan alcanzar los objetivos.

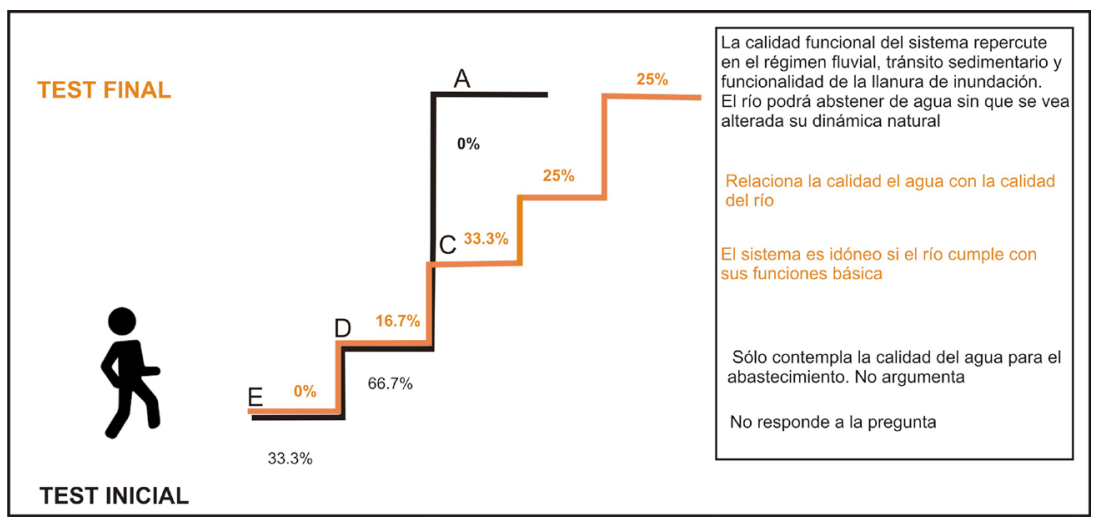

Figura 10. Aprendizaje sobre la pregunta 3a

TEST FINAL
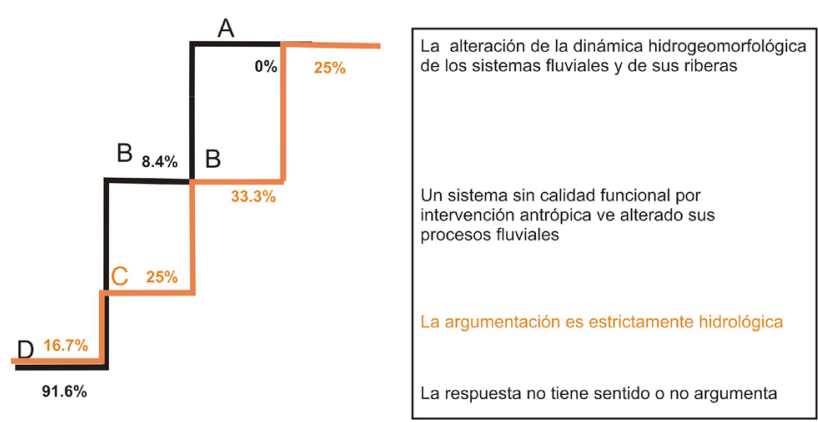

TEST INICIAL

Figura 11. Aprendizaje sobre la pregunta 3b

Respecto al aprendizaje individual se pone de manifiesto la existencia de dos tipos de aprendizaje: uno 
vinculado a la asistencia regular a clase y que sigue el ritmo que marca el docente (alrededor de 9-10 alumnos); y otro asociado a aquellos alumnos que no han asistido regularmente a clase y/o que llevan un ritmo más lento en el aprendizaje. A este segundo grupo se le está reforzando con tutorías personalizadas.

\section{Evaluación del Ciclo de Mejora Docente}

La implementación del CMD ha supuesto para mí un gran esfuerzo por concretar los contenidos articuladores de la materia. Reflexión que he de continuar haciendo, en un futuro próximo, para definir bien cuáles son los contenidos esenciales y cuáles no lo son tanto.

He de aprender que los tiempos son diferentes cuando uno trasmite el conocimiento que cuando el alumno ha de llegar a ellos a partir de documentos de contraste. Creo que esta ha sido una de las cuestiones que ha hecho que las actividades no hayan estado bien organizadas temporalmente, y que algunas tareas hayan tenido que realizarse entre sesión y sesión. A este respecto, es necesario reorganizar algunas sesiones e incorporar a las mismas un tiempo determinado para recoger el trabajo realizado por los alumnos.

Por lo general, los alumnos han mostrado una buena predisposición, aunque en algunas cuestiones, como por ejemplo, la definición de hipótesis previas, ha sido toda una conquista. Las reticencias y dificultades encontradas en las primeras sesiones se han ido desvaneciendo progresivamente, ya que los alumnos también necesitan gradualidad y transición en el cambio de rol. El alumno ha ido vinculándose con su aprendizaje a partir de su propia investigación y de los resultados que ha ido obteniendo. 
Ha sido una experiencia muy rica ya que he podido conocerme mejor como docente, tanto en las dificultades como en los éxitos que mis alumnos han ido consiguiendo en sus respectivos ámbitos de estudio. Para mi práctica habitual me quedo con el modelo metodológico posible de este ciclo de mejora, basado en problemas y/o proyectos, aún cuando es necesario incorporar el trabajo de campo como actividad de contraste.

Jornadas de Formación e Innovación Docente del Profesorado | № 1 (2018) Esta obra se distribuye con la licencia Creative Commons 


\section{Bibliografia}

Bain, K. (2004). What the Best College Teachers Do. Cambridge, MA. Harvard University Press (Trad. Cast.: Lo que hacen los mejores profesores universitarios. Valencia. Publicaciones de la Universidad de Valencia, 2007, 2a edición).

Duarte, O. (2015). "Los ciclos de mejora como hipótesis de progresión del modelo didáctico de investigación". En R. PORLÁn y E. NAVARRo (Coords.), III Jornadas de Docencia Universitaria (págs. 72-85). Sevilla. Instituto de Ciencias de la Educación de la Universidad de Sevilla.

Feria, AB (2017). "La Fisiología vegetal como ciencia integradora": una estrategia de enseñanza basada en la investigación". En R. PORLÁN (Coord.), Enseñanza universitaria. Cómo mejorarla (págs. 122-143) Sevilla, Ed. Morata S.L.

Finkel, D. (2000). Teaching with your mouth shut. Portsmouth, NH. Heinemann Boynton/ Cook. (Trad. Cast.: Dar clase con la boca cerrada. Valencia. Publicaciones de Universidad de Valencia, 2008)

García Díaz, E.; Porlán, R. y Navarro, E. (2017). "Los fines y los contenidos de enșeñanza" EN R. PORLÁN (Coord.), Enseñanza universitaria. Cómo mejorarla (págs.56-72) Sevilla, Ed. Morata S.L

Porlán, R.; Martín del Pozo, R.; Rivero, A, Harres, J.; Azcárate, P. y Pizarro, M. (2010). "El cambio "del profesorado de ciencias I: Marco teórico y formativo" Enseñanza de las Ciencias, 28(1), págs. 31-46.

Rivero, A. y Porlán, R. (2017). “La evaluación en la enseñanza universitaria".' En R. PORLÁN (Coord.), Enseñanza universitaria. Cómo mejorarla (págs., 74-91). Sevilla, Ed. Morata S.L.

Rivero, A.; Martín del Pozo, R.; Solís, E. y Porlán, R. (2017). Didáctica de las Ciencias Experimentales en Educación Primaria. Madrid. Sintesis. 